\title{
Advances in Muscle Physiology and Pathophysiology 2011
}

\author{
Aikaterini Kontrogianni-Konstantopoulos, ${ }^{1}$ Guy Benian, ${ }^{2}$ and Henk Granzier ${ }^{3}$ \\ ${ }^{1}$ Department of Biochemistry and Molecular Biology, University of Maryland, School of Medicine, Baltimore, MD 21201, USA \\ ${ }^{2}$ Departments of Pathology and Cell Biology, Emory University, Atlanta, GA 30322, USA \\ ${ }^{3}$ Departments of Physiology and Molecular and Cellular Biology, The University of Arizona, Tucson, AZ 85721, USA
}

Correspondence should be addressed to Aikaterini Kontrogianni-Konstantopoulos, akons001@umaryland.edu

Received 18 December 2011; Accepted 18 December 2011

Copyright ( 2012 Aikaterini Kontrogianni-Konstantopoulos et al. This is an open access article distributed under the Creative Commons Attribution License, which permits unrestricted use, distribution, and reproduction in any medium, provided the original work is properly cited.

The ordered assembly and synchronous contraction of striated muscle cells are highly complex processes that depend on the precise spatiotemporal formation of the sarcomeric and extrasarcomeric cytoskeleton alongside with specialized internal membranes that coordinately regulate $\mathrm{Ca}^{2+}$ cycling. The establishment of flexible molecular anchors that withstand stretch, between the contractile cytoskeleton and internal membranes, the superficial cytoskeleton and the sarcolemma, as well as the sarcolemma and the extracellular matrix, allows muscle cells to maintain their structural integrity and contractile activity. Recent advances using state-of-the-art, multidisciplinary, and integrated approaches have yielded a wealth of new information regarding these processes and have highlighted the next challenges for muscle physiologists, biochemists, and biophysicists.

In the second volume of our Advances in Muscle Physiology and Pathophysiology special emphasis issue, we continued to solicit original and review articles from leading and emerging scientists who study muscle structure and function and with diverse expertise and interests. A wide array of topics is covered, including, but not limited to, thin and thick filament assembly, regulation of cross-bridges formation, force generation, stress and stretch sensors, and $\mathrm{Ca}^{2+}$ dynamics as well as injury and disease models.

As the Editors of this special issue, we are grateful to all the authors who provided either original articles or comprehensive reviews of previous and current literature, making the second volume of this special issue attractive and interesting to a diverse audience of muscle researchers. Similar to our first volume, our aspiration was to include various aspects of muscle physiology and pathophysiology at the molecular, cellular, organ, and organismal levels. We are pleased to see the outcome of our call in the form of this second, special emphasis volume, and as before we expect that it will provide a wealth of information that will further inspire outstanding muscle research.

Aikaterini Kontrogianni-Konstantopoulos

Guy Benian

Henk Granzier 

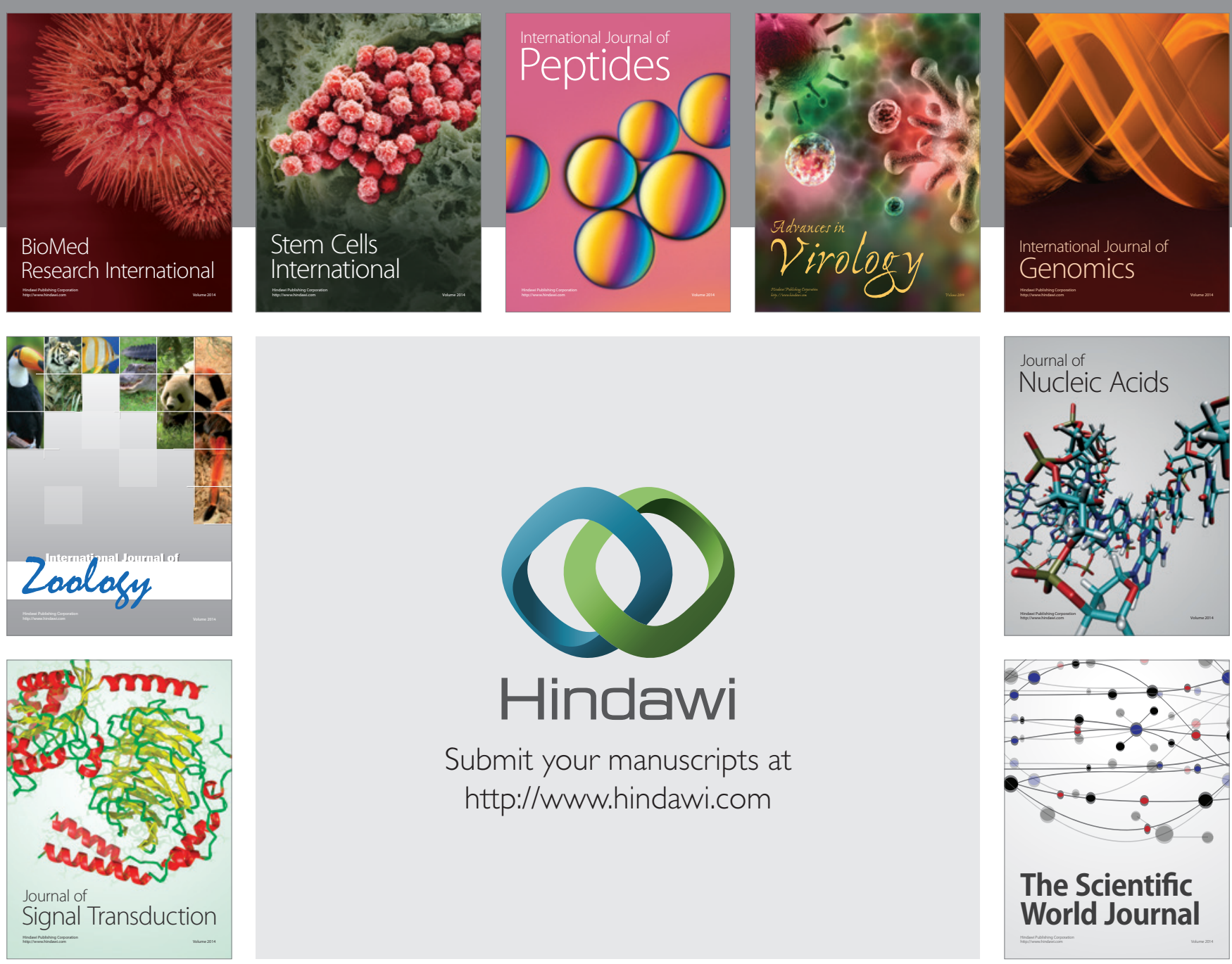

Submit your manuscripts at

http://www.hindawi.com
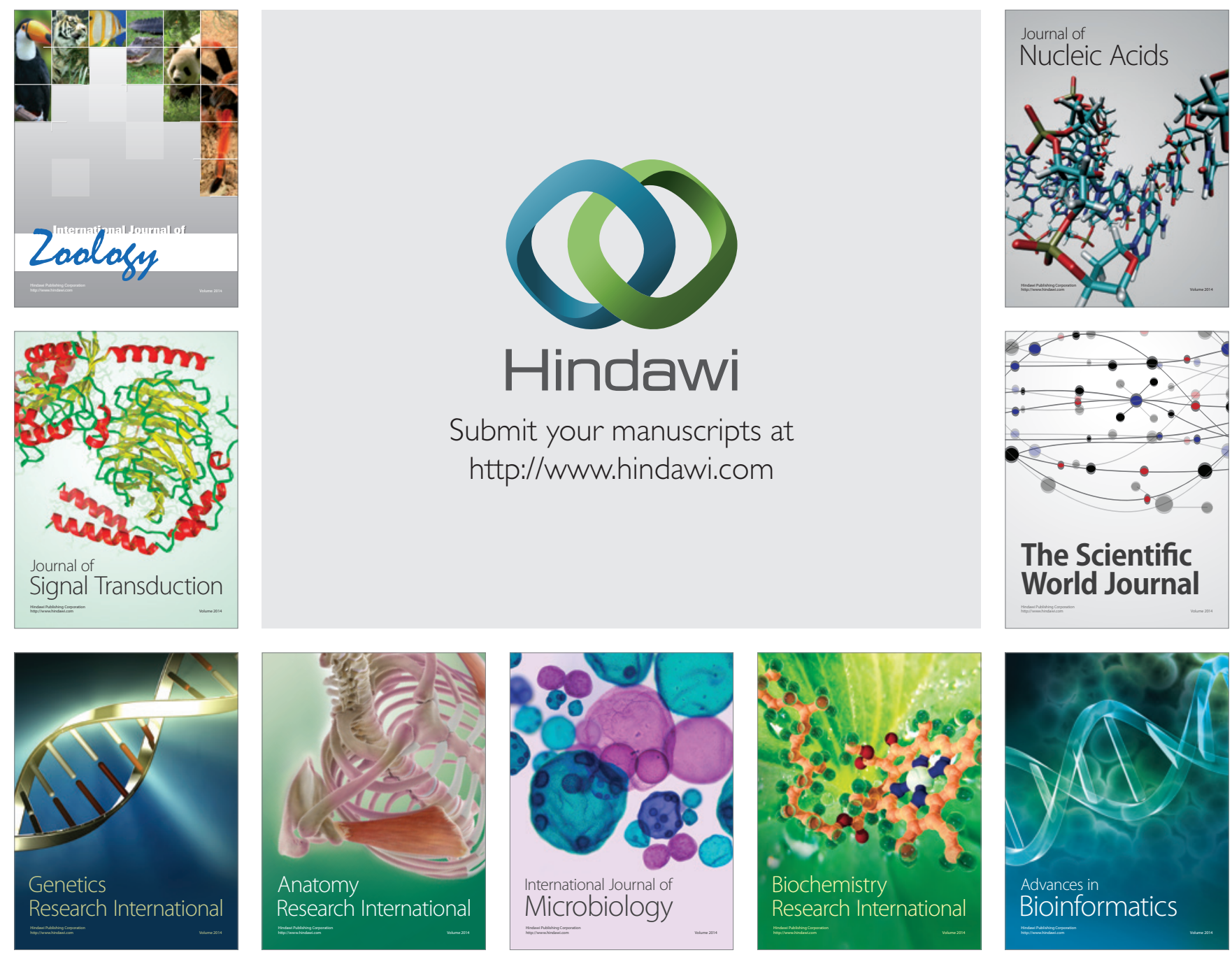

The Scientific World Journal
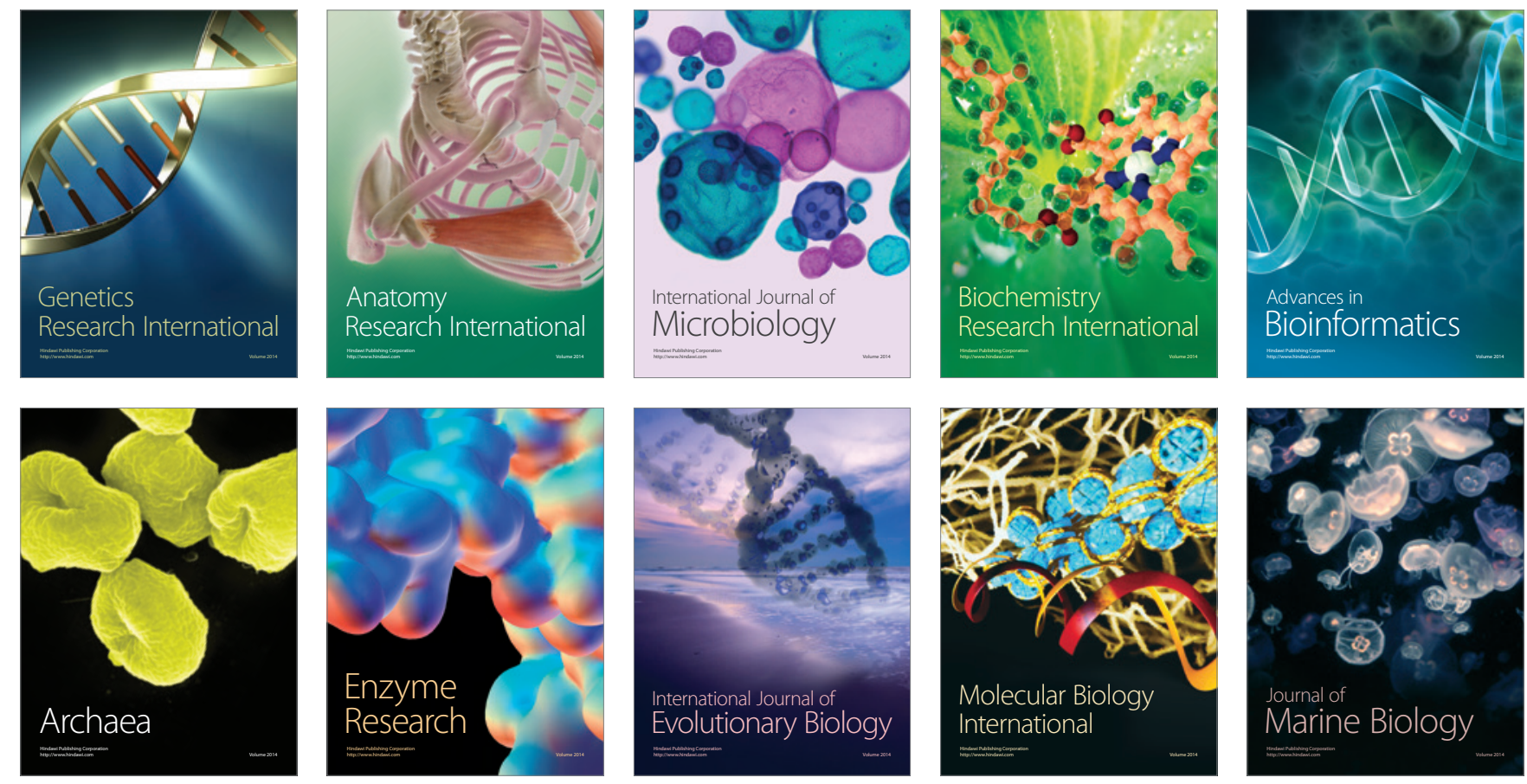